\title{
Synchronization or Coordination? Context dependent anti-predator vigilance to nature-based ecotourism
}

\author{
Dejun Kong ${ }^{1}$, Anders Møller ${ }^{2}$, and Yan-Yun Zhang ${ }^{3}$ \\ ${ }^{1}$ Kunming University \\ ${ }^{2}$ Université Paris-Saclay \\ ${ }^{3}$ School of Life Sciences, Beijing Normal University
}

July 16, 2020

\begin{abstract}
Prey monitor surrounding dangers independently or cooperatively (synchronized and coordinated vigilance), with independent and synchronized scanning being prevalent. Coordinated vigilance, including unique sentinel behaviour, is rare in nature, since it is time-consuming and benefit-limited. Evidence does not indicate animals adopting alternative vigilance strategies during antipredation scanning. Considering the cooperative nature of both synchronization and coordination, we assessed whether group members could keep alert synchronously or coordinatedly under different circumstances, determining whether cooperative vigilance is context dependent. Under the framework of conservation behavior, we studied how human behaviour and species-specific variables impacted individual and collective vigilance of globally threatened Black-necked Cranes (Grus nigricollis) and explored behaviour-based wildlife management. We tested both predation risk (juveniles in group) and human disturbance (level and distance) effects on individual and collective antipredation vigilance of black-necked crane families. Adults spent significantly more time (proportion and duration) on scanning than juveniles, and parents with juveniles behaved more vigilant. Observer distance affected individual vigilance of adults while juveniles were influenced by none of these variables. With the number of juveniles and disturbance increased, crane couples decreased synchronization of vigilance and they shifted to coordination, which has so far never been reported yet. Similarly, with observer approaching, adults shift vigilance from synchronization to coordination. The collective vigilance shift from synchronization to coordination as a function of observer distance could help us determining a safe distance of c. $400 \mathrm{~m}$ for the most vulnerable family groups with two juveniles, so as no obvious interference with the threatened birds by human proximity. We argue that vigilance behaviour could be a reliable indicator in future nature-based tourist management and decision-making, which can be derived from conservation solutions in nature.
\end{abstract}

\section{Synchronization or Coordination? Context dependent anti-predator vigilance to nature-based ecotourism}

Dejun Kong ${ }^{1,2, *}$, Anders Pape Møller ${ }^{2}, 3$, Yanyun Zhang ${ }^{2}$

1. Key Laboratory of Special Biological Resource Development and Utilization of Universities in Yunnan Province, College of Agronomy and Life Sciences, Kunming University, Kunming 650214, Yunnan, China.

2. Ministry of Education Key Laboratory for Biodiversity Science and Ecological Engineering, College of Life Sciences, Beijing Normal University, Beijing 100875, China.

3. Université Paris-Saclay, CNRS, AgroParisTech, Ecologie Systématique et Evolution, 91405, Orsay, France.

* Corresponding author: Dejun Kong, kongdj05@hotmail.com. 
Full postal address: No. 2 Puxin Road, Jingkai District, Kunming 650223, Yunnan, China.

Word number without Appendix: 5754.

\section{Highlights:}

1. Synchronization to coordinated vigilance was first reported from behavioural spectrum analysis in birds.

2. Synchronization of vigilance decreased and shifted as coordination increased with disturbance and predation risk.

3. Families with more juveniles were more vigilant.

4. Vigilance could constitute a prime indicator in behaviour-based tourist management.

5. We recommend to keep tourists at least a distance of $400 \mathrm{~m}$ away from cranes.

Abstract: Prey monitor surrounding dangers independently or cooperatively (synchronized and coordinated vigilance), with independent and synchronized scanning being prevalent. Coordinated vigilance, including unique sentinel behaviour, is rare in nature, since it is time-consuming and benefit-limited. Evidence does not indicate animals adopting alternative vigilance strategies during antipredation scanning. Considering the cooperative nature of both synchronization and coordination, we assessed whether group members could keep alert synchronously or coordinatedly under different circumstances, determining whether cooperative vigilance is context dependent. Under the framework of conservation behavior, we studied how human behaviour and species-specific variables impacted individual and collective vigilance of globally threatened Black-necked Cranes (Grus nigricollis ) and explored behaviour-based wildlife management. We tested both predation risk (juveniles in group) and human disturbance (level and distance) effects on individual and collective antipredation vigilance of black-necked crane families. Adults spent significantly more time (proportion and duration) on scanning than juveniles, and parents with juveniles behaved more vigilant. Observer distance affected individual vigilance of adults while juveniles were influenced by none of these variables. With the number of juveniles and disturbance increased, crane couples decreased synchronization of vigilance and they shifted to coordination, which has so far never been reported yet. Similarly, with observer approaching, adults shift vigilance from synchronization to coordination. The collective vigilance shift from synchronization to coordination as a function of observer distance could help us determining a safe distance of $c .400 \mathrm{~m}$ for the most vulnerable family groups with two juveniles, so as no obvious interference with the threatened birds by human proximity. We argue that vigilance behaviour could be a reliable indicator in future nature-based tourist management and decision-making, which can be derived from conservation solutions in nature.

Keywords: Anthropogenic disturbance; Collective vigilance; Ecotourism; Nature visitation; Predation risk.

\section{INTRODUCTION}

Human actions driver significant alternation to global nature systems since 1900, making dramatic biodiversity loss and approximately 1 million species face extinction, and unprecedented changes experienced by the nature during the past fifty years (IPBES, 2019). Anthropologic disturbance to wildlife not limited in human residential areas and nearby but also penetrated to protected areas through nature-based visitation and recreation (Geffroy et al., 2015; Marion et al., 2016; Slater et al., 2019), threating wildlife behaviour, abundance, reproduction (Steven et al., 2011) and increasing vulnerability of prey to predators (Geffroy et al., 2015). While conservationists believe that by through exploring indicator behaviours of wildlife, we could understand pressure wildlife experiencing from anthropogenic impacts, advance behavior-based management and achieve sustainable conservation and ecotourism (Berger-Tal et al., 2011; Blumstein et al., 2017). And one of those indicator behaviour is anti-predator vigilance (Berger-Tal et al., 2011).

Animals scan environments around to monitor potential threats from both predators and rivals, regarded as the behavior of vigilance (Caro, 2005; Beauchamp, 2015). Amounts of studies on antipredation behavior revealed that animals gather in large group to decrease individual vigilance, share collective vigilance benefit and avoid being captured (Pulliam, 1973). A great deal of these studies focused on individual vigilance based 
on the assumption that an individual initiate vigilance bout regardless of behavioral state of other members, named as independent vigilance (Pulliam, 1973; Caro, 2005). Cooperated vigilance behavior patterns of synchronization and coordination, however, were also observed in multiple animal systems when taking time origination of vigilance bout between group companions into consideration (Pays et al., 2007a, b).

Synchronized vigilance indicates individual copy neighbors' vigilance state by monitoring group companions (Pulliam, 1973) leading to collective vigilance wave (Beauchamp, 2010), also known as the allelomimetic vigilance (Quenette and Gerard, 1992; Pays et al., 2007a, b). Contagious vigilance in group may also induce collective waves of other activities e.g., foraging wave (Quenette and Gerard, 1992) and sleep wave (Beauchamp, 2010). Evidences from case studies and model analysis (Rodríguez-Gironés and Vásquez, 2002; Sirot and Touzalin, 2009) illustrate group members could often synchronize their vigilance in the field.

While coordinated vigilance refers to group member keeping vigilant alternatively in order to avoid scan gap of independent and synchronized vigilance by chance (Bednekoff, 2015). Sentinel behaviour, a wellknown form of coordinated vigilance was observed in limited range of vertebrates with cooperative breeding behaviour (Bednekoff, 2015), including mammals (Rasa, 1986; Clutton-Brock et al., 2013), birds (Wickler, 1985; Wright et al., 2001) and fish (Brandl and Bellwood, 2015). Since the likely small potential benefit in most cases, time paying in coordinated vigilance would be less worthy (Ward, 1985) and are seldom occurred in nature (Rodríguez-Gironés and Vásquez, 2002).

So, compared with group vigilance when each individual scan independently, collective vigilance with at least one member is vigilant should be expected higher in coordinated groups and lower in synchronized groups (Pays et al., 2007a, b). Up to date, three vigilance strategies were documented respectively in different study systems and no joint vigilance was reported yet. Ge et al (2011) reported synchronization of collective vigilance of two adult Red-crowned craneGrus japonesis in family groups decreased when birds shifted from core zone with less disturbance to buffer zone with higher disturbance; and common crane Grus grus behaved coordinated vigilance in buffer zones. Basically, smaller common crane showed stronger antipredation vigilance than bigger crane species (Kong et al., 2020), which means common crane could adopt elevated vigilance strategy to red-crowned crane facing similar threats or disturbance, e.g. the buffer zone disturbance in the study of Ge et al (2011). Then, regardless of species, we could make a rational inference that couple cranes may decrease vigilance synchronization with disturbance increase and shift to coordinated vigilance as predation risk or disturbance increase further in small groups (Wickler, 1985). So, we wonder whether synchronized and coordinated vigilance could be detected in nature, for a single species, concerning their common nature of cooperative vigilance and what factors drive the alternation of synchronization and coordination vigilance.

In this study, we tested (1) how environmental and group variables affect both individual and collective vigilance of black-necked cranes; (2) how they response to nature-based recreation through anti-predator vigilance adjusting and (3) moreover, we anticipated to determine an safety observation distance for both visitation and scientific research purpose from vigilance studying, which could also benefit conservation decision-making and management.

We examined the cooperative vigilance temporal pattern (synchronization and coordination) in an exclusively plateau distributed crane species, Black-necked Crane Grus nigricollis , an ideal candidate could be easily observed in distance for testing vigilance cooperation ( $\mathrm{Li}$ et al., 2017). The birds are facing direct interference from nature-based tourism and indirect threats from climate change and anthropogenic expansion induced wetland loss dramatically (Harris and Mirande, 2013; Li et al., 2014).

Since studies documented synchronization vigilance decreasing between group companies was driven by disturbance (Ge et al., 2011) and predation risk (Podgórski et al., 2016). Coordinated collective vigilance pattern, as a stronger antipredation response against disturbance (Ge et al., 2011) could also be observed for birds under strong disturbing circumstance while synchronized vigilance would be expected facing lower disturbance. We hypothesized that black-necked crane could decrease synchronized vigilance with disturbance and predation risk increase, even shift to coordinated vigilance, if possible. Concerning nature-based 
recreation in habitats of black-necked cranes are common, we take both disturbance level (categorial variable) and observer distance (continuous variable) into consideration. If vigilance alternation of synchronization to coordination could be detected indeed, then cooperated vigilance of black-necked cranes would vary as a function of the continuous variable of observer distance. Then we could regard the distance (intercept value with $\mathrm{X}$ axis) at which cranes alter vigilance from synchronized to coordinated as a control distance keeping tourist away from the birds; and this control distance could be valuable reference in future conservation aimed tourist management.

\section{MATERIALS AND METHODS}

\subsection{Study species}

Black-necked cranes are a vulnerable species in the IUCN Red List, endemic to the western plateaus of China (including the Qinghai-Tibet and Yunnan-Guizhou Plateau) with less than 5\% population inhabited in wetlands of Bhutan and India (Li, 2014). Breeding pairs occupy relative constant territories while nonbreeding sub-adults wander across vast areas in flocks (Zhang et al., 2017); they aggregate into flocks or in small family groups in winter (Liu et al., 2008). Family groups usually consist of parents and 0,1 or 2 juveniles. It is easy to distinguish juveniles from adults from blocky brownish feathers, smaller body size and whistles like calls. While adults have a distinct black neck, flight feathers and tail compared with white plumages. However, telling males from females apart is hardly achieved except from appearance of unison call.

\subsection{Study area}

This study was conducted in the Dashanbao National Nature Reserve (Dashanbao, N27'18'38 " -27deg29'15", E103deg14'55"-103deg23'49"), SW China. Covering an area of 19,200 ha, Dashanbao was first established in 1993 and upgraded to national level in 2003 for protecting the global threatened Black-necked Crane and plateau wetlands on which waterfowl depend. Dashanbao was famous not only for its role as an important wintering ground for black-necked cranes, but also a vital staging and stopover site for the eastern population of the bird (Kong et al., 2014b). Each year there are nearly 1,500 individuals wintering at Dashanbao, constituting $40 \%$ of their eastern population and $\sim 14 \%$ of the world population (Yang and Zhang, 2014). In 2004, Dashanbao was designated as a Ramsar wetland of international importance as the contribution in black-necked crane conservation. There are four roosting sites, named Dahaizi, Xiaohaiba, Changhuikou and Yinjiabeihaizi, located along the lakeside of Dashanbao reserve (Fig. 1). Dahaizi is the most famous site as supporting the largest number of 600-900 black-necked cranes each year (Kong et al., 2014b).

Gathering of the gorgeous cranes attracted massive influx of people there for bird-watching, photography and also landscape viewing. The local authority started nature-based tourism program in 2009. According to reports of Dashanbao administration bureau, over 10,000 tourists, more than 10 times before tourism was implemented, went to Dashanbao at the end of 2009, with a yearly increasing rate of $>30 \%$. Over 60,000 people were recorded in 2013-2014 (Yang and Liu, 2014), sixty times of increase in abundance after entrance permit to the public. Sharp increase in tourism caused great threats to the black-necked crane population and other wildlife there. As the Dashanbao ecotourism overall plan indicated four discrete areas (named Dahaizi lake area, Tiaodunhe lake area, Jigong mountain and valley area, and Yanmaidi lake area) opened to the public, where three of the four black-necked roosting sites were located in two of these areas (Figure 1). People went to Dashanbao visit Dahaizi Lake area (for crane observation and photograph) and Jigong mountain and valley area the most (Yang and Liu, 2014), occasionally they drove around the Tiaodun Lake, but seldom to Yanmaidi lake where cranes were occasionally present. Thus, cranes were distributed in the Dahaizi and Jigong area and along the roadside to these areas facing high levels of disturbance, and cranes in Tiaodunhe area are facing intermediate disturbance while cranes in the other areas encountered the lowest disturbance from local native people (Figure 1).

\section{INSERT FIGURE 1 HERE}

\subsection{Data collection}

We conducted behavioral observations of black-necked cranes during 09:00-18:00 in the winter 2013-2014. 
Cranes were firstly detected along a $96 \mathrm{~km}$ long line transect by vehicle (Figure 1). Once cranes were located one observer walked to the focal flock and collected behaviour data of the birds with a digital video recorder (Sony HDR-XR260E with 55x optical zoom) set on a tripod. Meanwhile distance to the birds was measured with a laser rangefinder monocular (Newcon Optik LRM 2200SI with a maximum range of 2,200 meters). We aimed to take 10 minutes (mins) film for each family group encountered, because it is sufficiently long to get sufficient vigilance bouts for black-necked cranes ( $\mathrm{Li}$ et al., 2017). In order to get time series data of each family member, we made sure each individual was in the picture at the same time; film recording ceased as long as one bird was out of sight or flew away (Ge et al., 2011). Vigilant and non-vigilant behavior were extracted in second (s) from videos taken in the field. We defined head-up scanning with neck stretched while standing, walking or even laying down as vigilance (Kong et al., 2020), otherwise as non-vigilant.

\subsection{Data processes}

All our filmed pictures are sufficiently stable to distinguish each behavioural state and long enough to meet at least 5 samples of vigilance/non-vigilance bouts for statistical effectiveness, even for our shortest video sampling of $2.2 \mathrm{~min}$, as scan and inter-scan duration for black-necked crane are $4 \mathrm{~s}$ and $20 \mathrm{~s}$ on average (Li et al., 2017). So, all our data were taken into subsequent analysis. Vigilance behaviour was evaluated by both vigilance proportion and duration for each group member. Vigilance proportion was calculated as percentage of time spent on vigilance during the observation secession while vigilance duration as the average time span (seconds) of each vigilance bout (Li et al., 2017). Studies documented that group members could coordinate individual vigilance in order to increase group collective vigilance (Rasa, 1986; Bednekoff, 2015), and in small crane groups (Ge et al., 2011). We focused on the collective vigilance time pattern (time serials) of two adults in family groups, as no vigilance interactions between juveniles and adults were detected (Ge et al, 2011; Che et al., 2018).

We classified the areas where cranes occurred into low, intermediate and high disturbance levels according to tourist accessibility. Cranes distributed along the road to Dahaizi lake and Jigong mountain where nearly $90 \%$ tourist visited facing the highest disturbance, moderate disturbance in the areas around Tiaodunhe lake with less $30 \%$ public visitation, and lowest disturbance in the other areas. We considered the observer (data collector) as proxy of potential tourists, the distance from observer to cranes is another effect variable on vigilance. Only one observer approached crane without aggressive behaving e.g., shouting, chasing, means the lowest disturbance from tourist. Because inexperience always correlated with high predation vulnerability, a higher proportion of juveniles in groups usually indicated higher predation risk (Xu et al., 2013; Beauchamp, 2015). We considered our study objectives of three family typs with two adults and 0-2 juveniles as three levels of predation risk; and adults without juveniles endure the lowest predation risk while families with two juveniles have the highest predation risk.

We found that our data sets deviated from normality in a one-way Kolmogorov-Smirnov test, and arcsine square root transformation for vigilance proportion and logarithmic transformation for vigilance duration were subsequently conducted in order to get normalized data for parametric tests. Comparisons of vigilance difference between adults and juveniles and observed to expected collective vigilance of two adults were accomplished with t-test. One-way ANOVA was used to test both individual and collective vigilance difference of adults in three family types. We selected general linear model to distinguish effects of disturbance level (categorical variable of three levels), observer distance (continuous variable) and predation risk (categorical variable of three family types) on both individual and collective vigilance deviation of black-necked crane (Ge et al., 2011). Collective vigilance deviation was used to determine collective vigilance pattern (synchronization or coordination) by considering the deviation between expected (independent vigilance) and observed collective vigilance (Pays et al., 2007a, b). Expected collective vigilance was calculated with the equation of $1-\left[\left(1-p_{1}\right) *\left(1-p_{2}\right)\right]$, where $p$ represents vigilance proportion of two adults in a family (Pays et al., 2007a, b; Ge et al., 2011). Statistics were accomplished with IBM SPSS 20.0 software with a two-tailed significant level of $\mathrm{p}<0.05$.

\section{RESULTS}




\subsection{Individual vigilance}

In total, we obtained 870 mins observations from 34 family groups. For each family the observation lasted for $10.2 \mathrm{mins}$ on average (range: 2.2-23.5 mins), which met our expectation of $10 \mathrm{~min}$.

Adults spent significantly more time on vigilance (adults: $27.30-31.28 \%$ vs juvenile: $8.74-16.65 \% ; p<0.05$ ) and relatively longer vigilant bout (adults: $17.4-12.8 \mathrm{~s} v$ juvenile: $8.4-11.8 \mathrm{~s} ; p>0.05$ ) than juveniles (Table $1)$. No significant difference in adult vigilance proportion $(F=0.106, d f=2, p=0.899)$ and duration $(F$ $=0.833, d f=2, p=0.440$ ) among three family groups existed.

Vigilance behavior of adults was closely related to observer distance (vigilance proportion: $F=3.139, d f$ $=24, p=0.001$; vigilance duration: $F=1.804, d f=24, p=0.053)$ while juvenile vigilance was not significantly correlated with any of the variables of family type, disturbance level and observer distance $(p<0.05$, Appendix table 1).

\section{INSERT TABLE 1 HERE}

\subsection{Collective vigilance of adults}

For the three family groups, there were no collective vigilance difference of adults $(F=0.382, d f=24, p$ $=0.685)$. Collective vigilance of adults was significantly related to interactive effects of observer distance and family type $(F=25.372, d f=1, p=0.037)$.

\subsection{Collective vigilance pattern}

The deviation of observed and expected collective vigilance did not differ significantly from zero $(t=-1.127, d f$ $=31, p=0.268$ ); and deviation between expected and observed collective vigilance did not differ significantly among family type $(F=1.940, d f=2, p=0.340)$, disturbance level $(F=0.699, d f=1, p=0.491)$ or observer distance $(F=0.740, d f=20, p=0.718)$.

However, we found, in family group, two adults behaving synchronized collective vigilance in low disturbance areas when juveniles were absent, synchronization decreased with increased disturbance and predation risk (more juveniles in group) to independent vigilance, and then to coordinated collective vigilance at high level of disturbance with two juveniles (Figures. 2, 3).

We also found that collective vigilance deviation varied as a function of observer distance, that crane couples shifted synchronously to coordinate vigilance with observer approach (Figure 4). On average, adults shifted collective vigilance from synchronization to coordination when observers were $400 \mathrm{~m}$ away for the most vulnerable family with two juveniles, while two adult birds with 0-1 juveniles kept synchronized vigilance (Figure 4). While black-necked cranes could endure human approach to about averagely $100 \mathrm{~m}$ at population level (Appendix Figure 1).

INSERT FIG. 2-5 HERE

\section{DISCUSSION}

\subsection{From synchronization to coordination}

With spectral analysis of vigilance behaviour that takes time sequences of vigilant and non-vigilant state into consideration, we reported the first evidence of group vigilance shift from synchronization to independence and to coordination. As expected from theory, coordination is evolutionarily unstable (Rodriguez-Girones and Vasquez, 2002; Sirot and Touzalin, 2009), less important time-expenditure (Ward, 1985), sentinels are at great risk of getting depredated (Ridley et al., 2013), unless significant benefit could be met to enhance survive and foraging odds (Santema and Clutton-Brock, 2013; Brandl and Bellwood, 2015) or to help coping with frequent disturbances (Ge et al., 2011) of family members within small groups (Wickler, 1985; Rasa, 1986; Rodriguez-Girones and Vasquez, 2002). Although coordinated vigilance is a kind of time-waste behaviour, it leaves fewer alert gaps for foraging groups (Pulliam, 1973; Sirot and Touzalin, 2009).

\subsection{Collective vigilance and affecting factors}


Our study indicated that cooperative vigilance shift was disturbance and predation risk dependent. Many studies demonstrated that cooperative sentinel activity (one form of coordinated vigilance) increase when pups are present (Santema and Clutton-Brock, 2013) and when predation risk becomes greater (Ridley et al., 2010; Rauber and Manser, 2017). These findings support our findings that cranes coordinate vigilance pattern when more young birds are present, in high disturbance areas and when humans show proximity. In our study, we considered the number of juveniles in a family as predation vulnerability or risk (Xu et al., 2013), as predators prefer hunting those left behind (Sirot and Touzalin, 2009), more juveniles cost more individuals and collective alert time of adults (Table 1). Leading parents to adopt time-consuming coordinated vigilance strategy (Figure 2) as our study indicated. Significant interactive effects of family type and observer distance on adult collective vigilance indicate that cranes with juveniles are more vulnerable to human disturbance. Our study also emphasizes that coordinated vigilance or sentinel behaviour may actually exist in relatively vast systems in small family groups (Ward, 1985; Wickler, 1985), under circumstances with significant disturbance and predation risk (Rauber and Manser, 2017), even for monogamous breeding systems (Mainwaring and Griffith, 2013) as in black-necked cranes.

\subsection{Discovering cooperative vigilance pattern by spectral analysis}

Different from a recent vigilance synchronization study on black-necked crane wintering at Lhasa, Tibet, Che et al. (2018) did not detect significant deviation between observed and expected collective vigilance. They concluded that no synchronized vigilance occurred for family members. However, it is difficult to figure out which cooperative anti-predator behavioural strategy group members adopted regardless of vigilance spectrum (Pays et al., 2007a, b). As our study indicated no significant difference between expected and observed collective vigilance of two adults, revealing independent scan of two adults. When compared collective vigilance deviation with zero (when collective vigilance deviation above the value of zero, coordination occurs; in contrast, synchronization occurs) as spectral analysis suggested (Pays et al., 2007a, b; Ge et al., 2011), we found parent cranes cooperating their vigilance bouts in different situations (Figures. 2-4). When the family is facing great threats e.g., more juveniles, higher disturbance and more proximity to human observers, adults shift collective scan from synchronous to asynchronous ways, so as to ensure less scan gap left (Pulliam, 1973; Sirot and Touzalin, 2009). Just as our results proved that families with 2 juveniles performed stronger vigilance than families with $0-1$ juvenile.

\subsection{Increased individual vigilance with tourism}

Our study confirmed former studies (Wang et al., 2009; Che et al., 2018) that adults took the responsibility of anti-predator scanning with significant higher alert time expenditure and duration. Wildlife decrease individual vigilance and benefit from foraging by gathering in big flocks (Pulliam, 1973; Kong et al., 2020), but interspecific conflicts also increase with group size (Pulliam, 1973; Caro, 2005), and juveniles are more vulnerable to conflicts. So, in winter, we could observe many family groups (2 adults with 1-2 juveniles) forage separately from other large groups (Liu et al., 2008), so as to keep $>80 \%$ of time in foraging for young birds (Wang et al., 2009). Whereas adults in family groups have to spent $\sim 6 \%$ more time than adults in large groups in vigilance instead of foraging (Wang et al., 2009). Interestingly, in order to conquer high predation risk faced by family groups, both individual vigilance sequence organization and inter-scan intervals of blacknecked cranes are randomly distributed throughout the year ( $\mathrm{Li}$ et al., 2017), making vigilance bouts more unpredictable than predators could not initiate successful attack. Compared with other wintering grounds of black-necked cranes in China, including those at Caohai wetlands in Guizhou province (vigilance: 12.5916.52\%; Li and Ma, 1992), Napahai wetlands in Yunnan (vigilance: 11.74-17.05\%) and Lhasa in Tibet (vigilance: 17.8-21.0\%; Che et al., 2018), black-necked cranes in Dashanbao spent most time allocation to vigilance (27.30-31.28\%); even higher than former research conducted here in 2008 (vigilance: 15.6-21.4\%; Kong et al., 2008) before visitation opens for public tourists. Black-necked cranes in our study area are facing equivalent disturbance to the birds in Daqiao wetland (vigilance: $27.9+-16.5 \%$; Kong et al., 2020) with a large human density of 135 residents per square kilometers, about 80km away from Dashanbao. Similarly, individual vigilance of cranes in our study (observer distance) and in Daqiao are significantly affected by human disturbance (Kong et al., 2020). We believe that the predominant landscape of vast marshes in 
Napahai and Caohai wetlands, which the help of avoidance of human access to crane habitats contributed the lower anti-predator scans. Wintering habitats utilized by cranes in Dashanbao and Daqiao are farmland (Kong et al., 2018). From a historical view, our study also demonstrated that the explosive expands of nature-based tourism cause new threats to the threatened black-necked cranes (Li et al., 2014). So, we argue that it is likely that there is strong interference from tourism caused coordinated vigilance in black-necked cranes in our study.

\subsection{Conservation and management implications}

In our study, we found that collective vigilance of two adults varied as a linear function of observer distance, which helps us determining a distance at which cranes change vigilance from synchronized to coordinated behaviour. As vigilance of synchronization usually occurred under lower disturbance or predation risk circumstance as our results and former studies indicated, we named this distance from observer to black-necked cranes the least safe distance. From the function, we quantified the least safe distance for black-necked cranes and most vulnerable group of $2-2$ family was $c$. $100 \mathrm{~m}$ and $400 \mathrm{~m}$ respectively. The $100 \mathrm{~m}$ safe distance for black-necked crane population is very close to the flight initiation distance (FID) of $76 \mathrm{~m}$ for the bird (our unpublished data). Since cranes benefit from large group sizes (Pays et al., 2007a), 2-2 families actually were the most vulnerable group for black-necked cranes. Then, for the black-necked crane, we suggest to keep at least $400 \mathrm{~m}$ distance from tourists in order to create the lowest disturbance, which is valuable in conservation and management. Moreover, our study lets us determine the solution in nature.

AUTHOR CONTRIBUTION: D. Kong: Conceptualization, Methodology, Formal analysis, Original draft preparation, Reviewing and Editing. A.P. Moller: Writing, Reviewing and Editing. Y. Zhang: Software, Formal analysis, Writing, Reviewing.

FUNDING: This work was supported by the National Natural Science Foundation of China (Grant number: $31201725,31760624)$. The funders had no role in study design, data collection and analysis, preparation of the manuscript, or decision to publish.

DECLARATIONS : All authors declare that they have no competing interests.

DATA AVAILABILITY STATEMENT : All datasets in the present study are available at Dryad doi: 10.5061/dryad.5mkkwh73j.

\section{ACKNOWLEDGMENT}

We thank staff from the Dashanbao National Nature Reserve for their field assistance. Many thanks to Mr. Xibao Chen, Dongming Li and Miss Yanxu Dong who helped compiling behavioural data. We would like to thank Dr. Wanting Peng for her assistance in map construction and drafting comments.

\section{REFERENCES}

Beauchamp G. 2010. Collective waves of sleep in Gulls (Larus spp. ). Ethology, 117: 326-331.

Beauchamp G. 2015. Animal vigilance: Monitoring predators and competitors. San Diego, CA: Academic Press.

Bednekoff PA. 2015. Sentinel behavior: a review and prospectus. Advances in the Sstudy of Bbehavior, 47: 115-145.

Berger-Tal O, Polak T, Oron A, Lubin Y, Kotler BP, Saltz D. 2011. Integrating animal behavior and conservation biology: a conceptual framework. Behavioral Ecology, 22(2): 236-239.

Blumstein et al., eds. 2017. Ecotourism's promise and peril: A biological evaluation. Berlin: Springer.

Brandl SJ, Bellwood DR. 2015. Coordinated vigilance provides evidence for direct reciprocity in coral reef fishes. Scientific Reports, 5:14556. doi: 10.1038/srep14556.

Caro TM. 2005. Antipredator defenses in birds and mammals. Chicago: University of Chicago Press. 
Che Y, Yang L, Li ZQ. 2018. Vigilance synchronization of wintering black-necked crane (Grus nigricollis ) families in Tibet. Acta Ecologica Sinica, 38(4): 1375-1381.

Clutton-Brock, T. H., O’Riain, M. J., Brotherton, P. N. M.,Gaynor, D., Kansky, R., Griffin, A. S., Manser, M. 1999. Selfish sentinels in cooperative mammals. Science, 284: 1640-1644.

Geffroy B, Samia DSM, Bessa E, Blumstein DT. 2015. How nature-based tourism might increase prey vulnerability to predators. Trends in Ecology and Evolution, 30(12): 755-765.

Harris J, Mirande C. 2013. A global overview of cranes: status, threats and conservation priorities. Chinese Birds 4(3):189-209.

IPBES. 2019. Summary for policymakers of the global assessment report on biodiversity and ecosystem services of the Intergovernmental Science-Policy Platform on Biodiversity and Ecosystem Services. S. Diaz, J. Settele, E. S. Brondizio E.S., H. T. Ngo, M. Gueze, J. Agard, A. Arneth, P. Balvanera, K. A. Brauman, S. H. M. Butchart, K. M. A. Chan, L. A. Garibaldi, K. Ichii, J. Liu, S. M. Subramanian, G. F. Midgley, P. Miloslavich, Z. Molnar, D. Obura, A. Pfaff, S. Polasky, A. Purvis, J. Razzaque, B. Reyers, R. Roy Chowdhury, Y. J. Shin, I. J. Visseren-Hamakers, K. J. Willis, and C. N. Zayas (eds.). IPBES secretariat, Bonn, Germany.

Kong DJ, Luo WX, Liu Q, Li ZQ, Huan GY, Zhang JJ, Yang XJ. 2018. Habitat use, preference, and utilization distribution of two crane species (Genus: Grus ) in Huize National Nature Reserve, YunnanGuizhou Plateau, China. PeerJ, 6: e5105.

Kong DJ, Luo WX, Moller AP, Zhang YY, Yang XJ. 2020. Vigilance strategy differentiation between sympatric threatened and common crane species. Behavioural Processes, 176: 104119. doi: 10.1016/j.beproc.2020.104119.

Kong DJ, Yang XJ, Liu Q, Zhong XY, Yang JX. 2008. Diurnal time budget and behavior rhythm of wintering black-necked crane (Grus nigricollis ) at Dashanbao in Yunnan. Zoological Research 29(2):195-202.

Kong DJ, Zhong XY, Yang JX, Yang XJ. 2014. Seasonal and daily migration behavior of Black-necked Cranes (Grus nigricollis ) in Yunnan, China: timing and volume. Zoological Research, 35(S1): 181-188.

Li FS, Ma JZ. 1992. Wintering time budget and benefit study between family and group Black-necked Cranes. Chinese Wildlife, 3: 36-41,29.

Li FS. 2014. IUCN Black-necked Crane (Grus nigricollis ) conservation plan. Zoological Research, 35(S1):39 .

Li ZQ, Che Y, Yang L. 2017. Can sequential vigilance be predicted? Behavioural Processes, 145: 81-85.

Liu Q, Yang XJ, Zhu JG, Zhao JL, Yu HZ. 2008. Flock of Black-necked cranes wintering at Napahai nature reserve, China. Zoological Research, 29(5): 553-560.

Mainwaring MC, Griffith SC. 2013. Looking after your partner: sentinel behaviour in a socially monogamous bird. PeerJ, 1:e83. doi: 10.7717/peerj.83.

Marion JL, Leung YF, Eagleston H, Burroughs K. 2016. A review and synthesis of recreation ecology research findings on visitor impacts to wilderness and protected natural areas. Journal of Forestry-Washington, 114(3): 352-362.

Pays O, Jarman PJ, Loisel P, Gerard JR. 2007a. Coordination, independence or synchronization of individual vigilance in the eastern grey kangaroo? Animal Bbehaviour, 73: 595-604.

Pays O, Renaud PC, Loisel P, Petit M, Gerard JF, Jarman PJ. 2007b. Prey synchronize their vigilant behaviour with other group members. Proceedings of the Royal Society B, 274: 1278-1291.

Podgorski T, Jong S, Bubnicki JW, Kuijper DPJ, Churski M, Jędrzejewska B. Drivers of synchronized vigilance in wild boar groups. Behavioural Ecology, 27(4): 1097-1103. 
Pulliam HR. 1973. On the advantages of flocking. Journal of theoretical Biology, 38: 419-422.

Quenette, P. Y., Gerard, J. F. 1992. From individual to collective vigilance in wild boars (Sus scrofa). Canadian Journal of Zoology, 70, 1632-1635.

Rasa OAE. 1986. Coordinated vigilance in Dwarf Mongoose family groups: the "Watchman's song" Hypothesis and the costs of guarding. Ethology, 71: 340-344.

Rauber R, Manser MB. 2017. Discrete call types referring to predation risk enhance the efficiency of the meerkat sentinel system. Scientific Rereports, 7:44436. doi: 10.1038/srep44436.

Ridley AR, Nelson-Flower MJ, Thompson AM. 2013. Is sentinel behaviour safe? An experimental investigation. Animal Behaviour, 85: 137-142.

Ridley AR, Raihani NJ, Bell MBV. 2010. Experimental evidence that sentinel behaviour is affected by risk. Biology Letters, 6(4): 445-448.

Rodríguez-Gironés MA, Vásquez RA. 2002. Evolutionary stability of vigilance coordination among social forages. Proceedings of the Royal Society B, 269: 1803-1810.

Santema P, Clutton-Brock T. 2013. Meerkat helpers increase sentinel behaviour and bipedal vigilance in the presence of pups. Animal Behaviour, 85: 655-661.

Sirot E, Touzalin F. 2009. Coordination and synchronization of vigilance in groups of prey: the role of collective detection and predators' preference for stragglers. The American Naturalist, 173(1): 47-59.

Slater C, Cam G, Qi Y, Liu Y, Guay PJ, Weston MA. 2019. Camera shy? Motivations, attitudes and beliefs of bird photographers and species-specific avian responses to their activities. Biological Conservation, 237: 327-337.

Steven R, Pickering C, Castley JG. 2011. A review of the impacts of nature-based recreation on birds. Journal of Environmental Management, 92: 2287-2294.

Wang K, Yang XJ, Zhao JL, Yu HZ, Min L. 2009. Relations of daily activity patterns to age and flock of wintering black-necked crane at Napa Lake, Shangri-La in Yunnan. Zoological Research, 30(1): 74-82.

Ward PI. 1985. Why birds in flocks do not coordinate their vigilance periods. Journal of Theoretical Biology, 114, 383-365.

Wickler W. 1985. Coordination of vigilance in bird groups: the "watchman's song" hypothesis. Z. Tierpsychol., 69: 250-253.

Wright J, Berg E, De Kort SR, Khazing V, Maklakov AA. 2001. Cooperative sentinel behaviour in the Arabian babble. Animal Bbehaviour, 62: 973-979.

Xu, F., Ma, M., Yang, W.K., Blank, D., Ding, P., Zhang, T., 2013. Vigilance in Black-necked Cranes: effects of predation vulnerability and flock size. The Journal of Wilson Ornithology 125 (1), 208-212.

Zhang LX, An B, Shu ML, Yang XJ. 2017. Nest-site selection, reproductive ecology and shifts within core-use areas of Black-necked Cranes at the northern limit of the Tibetan Plateau. PeerJ 5: e2939. DOI $10.7717 /$ peerj.2939.

\section{TABLES AND FIGURES}

TABLE 1. Vigilance (proportion and duration) difference between adults and juveniles in three family groups of Grus nigricollis. The first figure for each family type is the number of adult and the latter is the number of juveniles in a family group. Bold figures indicate significant level of $p<0.05$.

\begin{tabular}{|c|c|c|c|c|c|c|}
\hline Family types & Age & $\mathrm{N}$ & Vigilance proportion & Vigilance proportion & Collective vigilance & Vigilance $\mathrm{d}$ \\
\hline & & & Mean \pm SD & T-test & Mean \pm SD & Mean $\pm \mathrm{SD}$ \\
\hline
\end{tabular}




\begin{tabular}{lllllll}
\hline Family types & Age & $\mathrm{N}$ & Vigilance proportion & Vigilance proportion & Collective vigilance & Vigilance d \\
\hline F2-0 & Adults & 12 & $28.29 \pm 25.15 \%$ & - & $34.03 \pm 30.51 \%, \mathrm{n}=10$ & $17.36 \pm 29.5$ \\
$\mathrm{~F} 2-1$ & Adults & 33 & $27.30 \pm 20.00 \%$ & $\mathrm{t}=2.212, \mathrm{df}=47, \mathbf{p}=\mathbf{0 . 0 3 2}$ & $43.92 \pm 32.18 \%, \mathrm{n}=16$ & $12.05 \pm 14.3$ \\
& Juveniles & 16 & $16.65 \pm 16.69 \%$ & & - & $11.78 \pm 10.8$ \\
$\mathrm{~F} 2-2$ & Adults & 21 & $31.28 \pm 21.45 \%$ & $\mathrm{t}=4.354, \mathrm{df}=32.024, \mathbf{p}=\mathbf{0 . 0 0 0}$ & $36.53 \pm 26.89 \%, \mathrm{n}=11$ & $12.81 \pm 10.2$ \\
& Juveniles & 20 & $8.74 \pm 8.72$ & & - & $8.41 \pm 5.70$ \\
Total & Adults & 66 & $28.75 \pm 21.18 \%$ & $\mathrm{t}=4.711, \mathrm{df}=100, \mathbf{p}=\mathbf{0 . 0 0 0}$ & $39.05 \pm 29.74 \%, \mathrm{n}=37$ & $13.26 \pm 16.8$ \\
& Juveniles & 36 & $12.26 \pm 13.29 \%$ & & - & $9.91 \pm 8.40$ \\
\hline
\end{tabular}

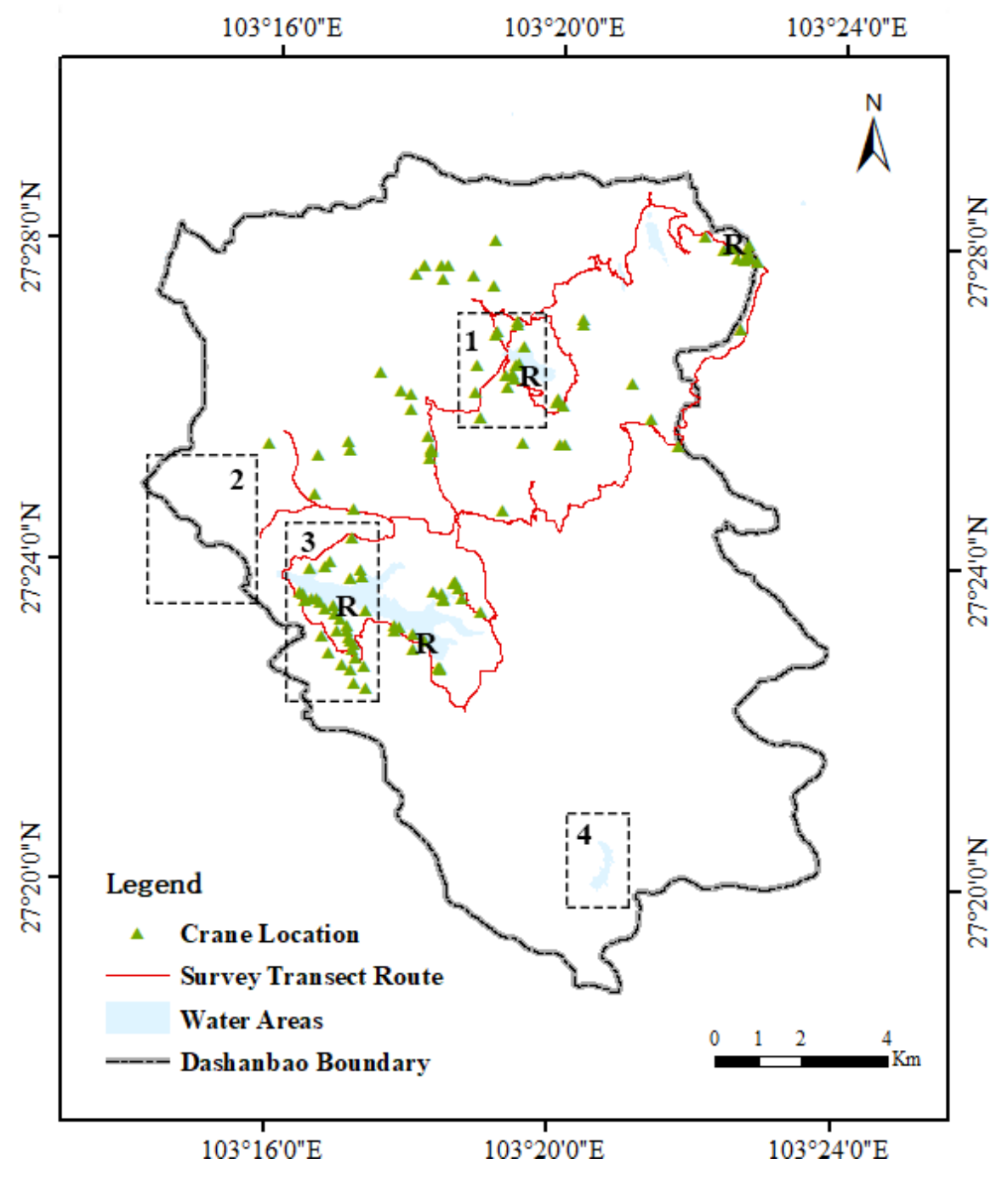

FIGURE 1. Study area of Dashanbao National Nature Reserve, Yunnan, SW China. The four dash line regulars numbered 1-4 is four area visited by tourists named Dahaizi lake area, Jigong mountain and valley area, Tiaodunhe lake area, and Yanmaidi lake area respectively. "R" indicates roosting sites. 


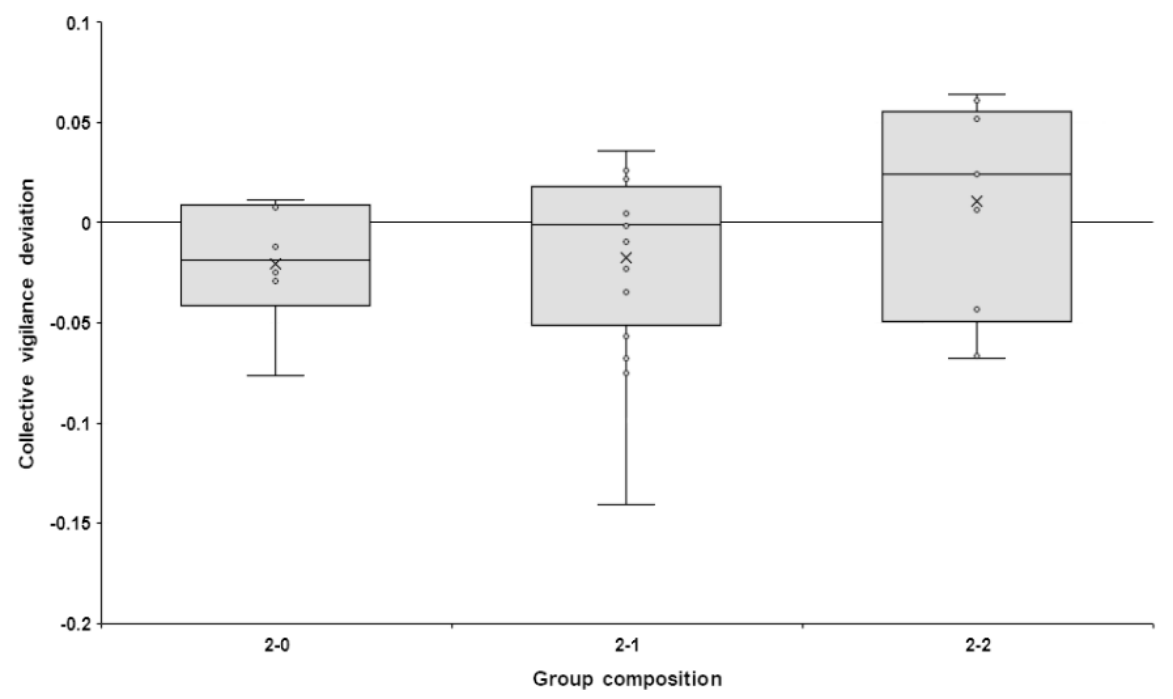

FIGURE 2. Comparison of observed and expected collective vigilance of two adults in three family groups. The figure of "2" in group composition indicates two adults in a family group and the latter figure of "0-2" indicates juvenile number in a family. Positive value of collective vigilance deviation means coordinated vigilance while negative is synchronized vigilance. The box plots show median, quartiles 5 - and 95- percentiles.

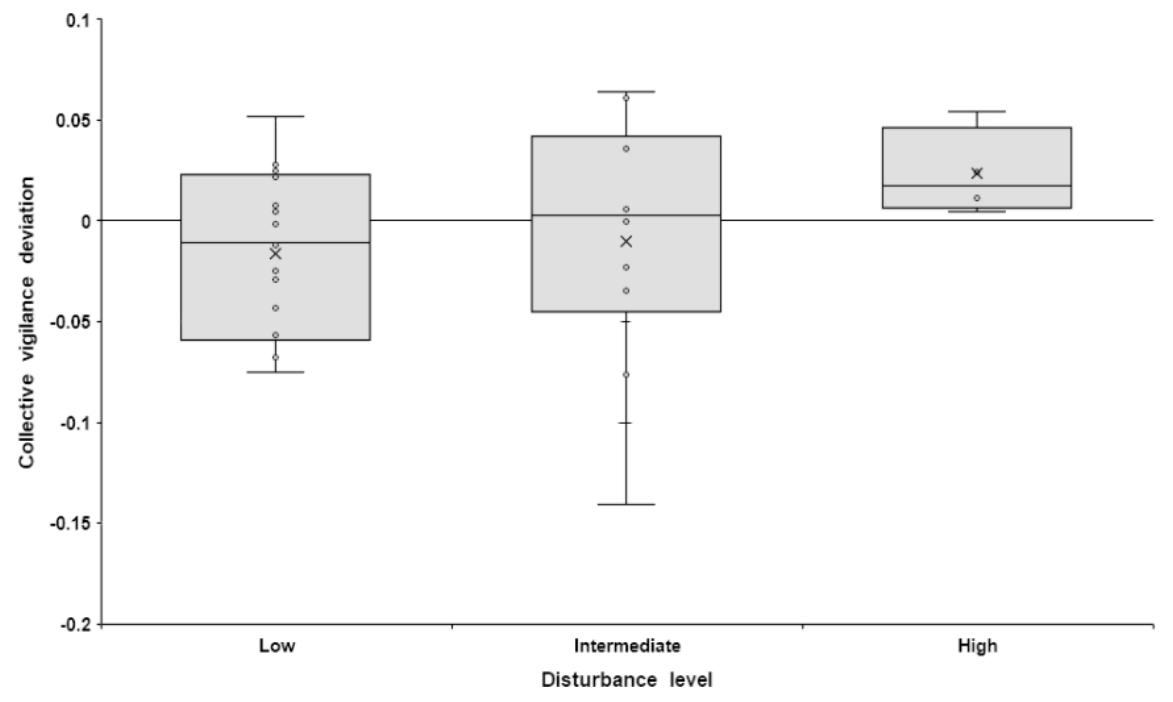

FIGURE 3. Comparison of observed and expected collective vigilance of two adults under different disturbance. Positive value of collective vigilance deviation means coordinated vigilance while negative is synchronized vigilance. The box plots show median, quartiles 5- and 95- percentiles. 


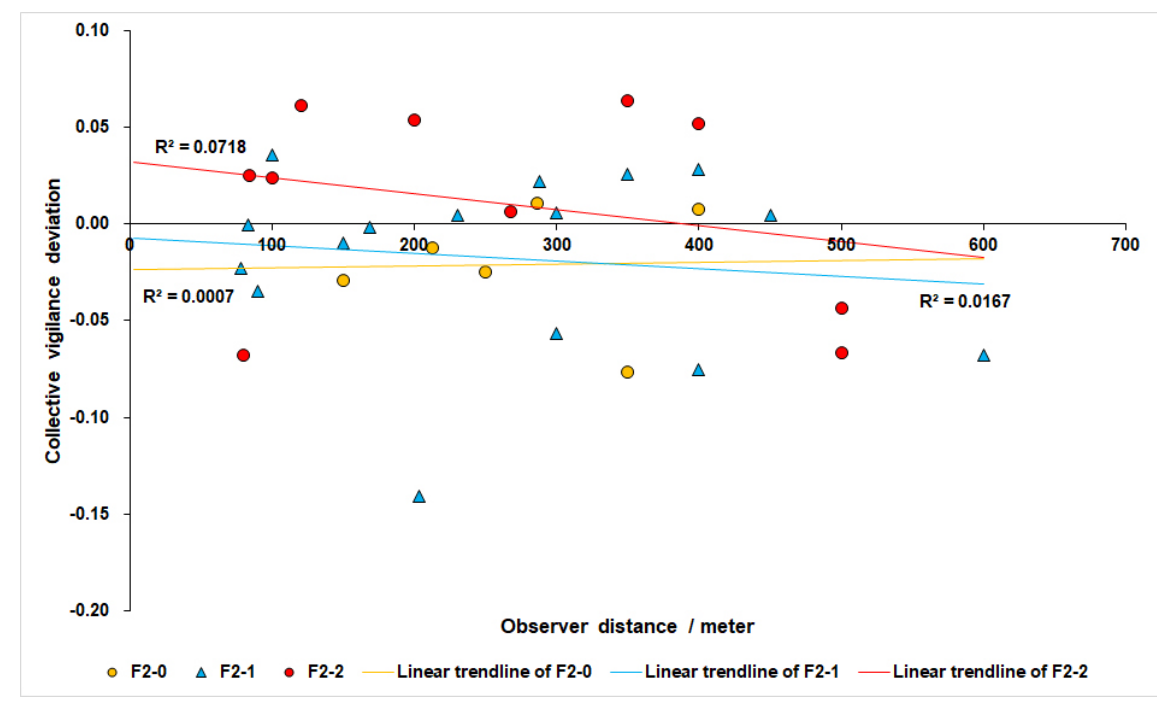

Fig. 4 Collective vigilance function of two adults in family groups to observer distance. Positive value of collective vigilance deviation means coordinated vigilance while negative is synchronized vigilance. F2-0, F2-1 and F2-2 indicate family groups of two adults with 0-2 juveniles.

\section{SUPPORTING INFORMATION}

APPENDIX TABLE 1. General linear model outputs of effect variables on individual vigilance of adults and juveniles.

\begin{tabular}{|c|c|c|c|c|c|c|c|}
\hline Response variables & Effect variables & Adults & Adults & Adults & Juveniles & Juveniles & $\overline{\text { Juveniles }}$ \\
\hline & & $\mathrm{df}$ & $\mathrm{F}$ & $\mathrm{P}$ & $\mathrm{df}$ & $\mathrm{F}$ & $\mathrm{P}$ \\
\hline \multirow[t]{4}{*}{ Vigilance proportion } & flock & 1 & 0.051 & 0.823 & 1 & 0.046 & 0.833 \\
\hline & disturbance & 2 & 0.216 & 0.807 & 2 & 2.517 & 0.122 \\
\hline & distance & 24 & 3.139 & 0.001 & 19 & 1.038 & 0.487 \\
\hline & disturbance ${ }^{*}$ distance & 1 & 0.754 & 0.391 & 1 & 2.678 & 0.128 \\
\hline \multirow[t]{4}{*}{ Vigilance duration } & flock & 1 & 0.408 & 0.527 & 1 & 1.526 & 0.245 \\
\hline & disturbance & 2 & 0.382 & 0.685 & 2 & 1.012 & 0.398 \\
\hline & distance & 24 & 1.804 & 0.053 & 19 & 1.775 & 0.177 \\
\hline & disturbance ${ }^{*}$ distance & 1 & 0.196 & 0.660 & 1 & 0.097 & 0.762 \\
\hline
\end{tabular}




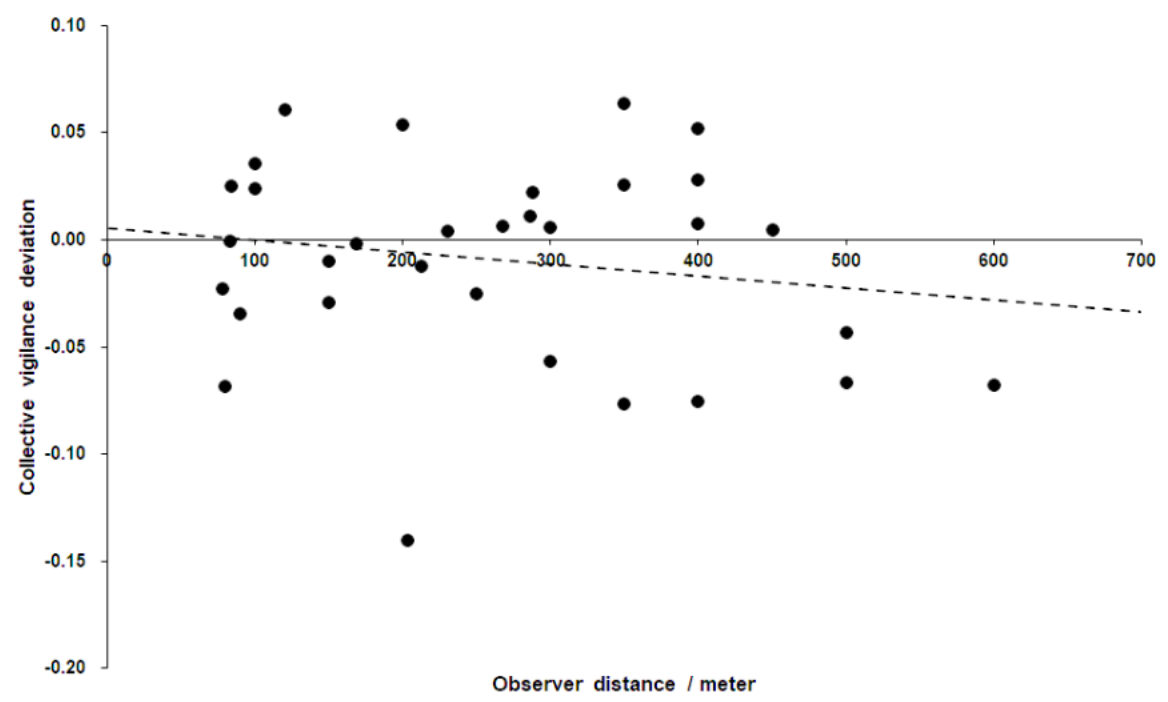

APPENDIX FIGURE 1. Collective vigilance function of two adults in family groups with two juveniles to observer distance. Positive value of collective vigilance deviation means coordinated vigilance while negative is synchronized vigilance. 